\title{
NNLO HTL QCD thermodynamics at finite temperature and chemical potentiale
}

\section{Najmul Haque*†}

Department of Physics, Kent State University, Kent, OH 44242, United States

In this proceedings contribution we present recent results of the thermodynamic potential at finite temperature and chemical potential for a system of quarks and gluons in hard-thermal-loop perturbation theory (HTLpt) at three-loop level. We compare the resulting pressure, energy density, trace anomaly at zero as well as at finite chemical potential and also second and fourth order diagonal and off-diagonal quark number susceptibilities with available lattice data. We show that there is good agreement between our three-loop HTLpt analytic results and available lattice data.

7th International Conference on Physics and Astrophysics of Quark Gluon Plasma

1-5 February, 2015

Kolkata, India

\footnotetext{
*Speaker.

${ }^{\dagger}$ Email: nhaque@kent.edu
} 


\section{Introduction}

In recent years, a lot of efforts have been undertaken to study the physics of quark gluon plasma (QGP) after the advent of modern heavy ion collider. From the last couple of decades, nonperturbative methods like lattice QCD (LQCD) simulations are very much successful to study and to explain various properties of QGP. Another alternative framework to study the various properties of QGP is perturbative method. One longstanding question is, to what extent can one use ideas stemming from perturbation theory for QGP phenomenology. One focal point in this regard has been high loop-order calculations of the QCD equation of state at finite temperature and density and comparison of these approximations to lattice QCD results. The perturbative expansion of the pressure of QCD at both zero [1] and non-zero chemical potential [2] are now known through order $g^{6} \ln g$. However, one finds in practice that a strict perturbative expansion in the running coupling converges only for temperatures many orders of magnitude higher than those relevant for heavyion collision experiments. The source of the poor convergence comes from contributions from soft momenta, $p \sim g T$. This suggests that one should treat the soft sector non-perturbatively, or at least reorganized the pertrbative series to treat the soft sector carefully.

There are various ways of reorganized the perturbative series at finite temperature and/or finite chemical potential. Hard-thermal-loop perturbation theory (HTLpt) is one of such methods which treats the soft sector more carefully. For scalar field theories one can resum pertrbative series using a simpler variant called "screened perturbation theory" (SPT) $[3,4,5,6]$. HTLpt, a gaugeinvariant generalization of SPT, was developed by Andersen, Braaten, and Strickland over a decade ago [7] using the concept of Hard Thermal Loops approximation by Braaten and Pisarski [8]. Since then HTLpt has been used to calculate various quantities viz. thermodynamic functions at one loop order [7, 9, 10,11], two loop order [12, 13, 14], and three loop order at zero chemical potential $[15,16,17]$ as well as at finite chemical potential(s) $[18,19]$.

In this proceedings contribution we present a recent calculation of the thermodynamic potential at finite temperature and chemical potential(s) to three-loop order (next-to-next-to-leading order or NNLO) in HTLpt. The result for equal quark chemical potentials was first presented in Ref. [18] and the extension to flavor-dependent chemical potentials was presented in Ref. [19]. In both cases, the resulting three-loop thermodynamic potential is renormalized using only known vacuum, mass, and coupling constant counterterms and the final result is completely analytic and gauge invariant. The resulting analytic thermodynamic potential can be used to obtain various thermodynamic quantities, viz., the pressure, energy density, entropy density, trace anomaly, speed of sound, and various quark number susceptibilities. As we will show below, there is good agreement between our NNLO HTLpt results and lattice data down to temperatures on the order of $250 \mathrm{MeV}$. Below we present plots of some of our main results and refer the reader to Ref. [19] for the calculation details and a more detailed discussion of the systematic uncertainties, etc.

\section{Results}

In this section we present some of the final results from Ref. [19]. For all results shown here we used the one-loop running coupling. We fixed the scale $\Lambda_{\overline{\mathrm{MS}}}=176$ for one loop running from the lattice measurements [20] by requiring that $\alpha_{s}(1.5 \mathrm{GeV})=0.326$. We use two separate 
renormalization scales, $\Lambda$ and $\Lambda_{g}$, for (pure and mix) fermionic feynman graphs and purely-gluonic, respectively. We take the central values of these renormalization scales to be $\Lambda_{g}=2 \pi T$ and $\Lambda=$ $2 \pi \sqrt{T^{2}+\mu_{q}^{2} / \pi^{2}}$. In all plots the thick black lines indicate the result obtained using these central values and the light-blue band indicates the variation of the result under variation of both of these scales by a factor of two, e.g. $\pi T \leq \Lambda_{g} \leq 4 \pi T$. For all numerical results below we use $N_{c}=3$ and $N_{f}=3$.
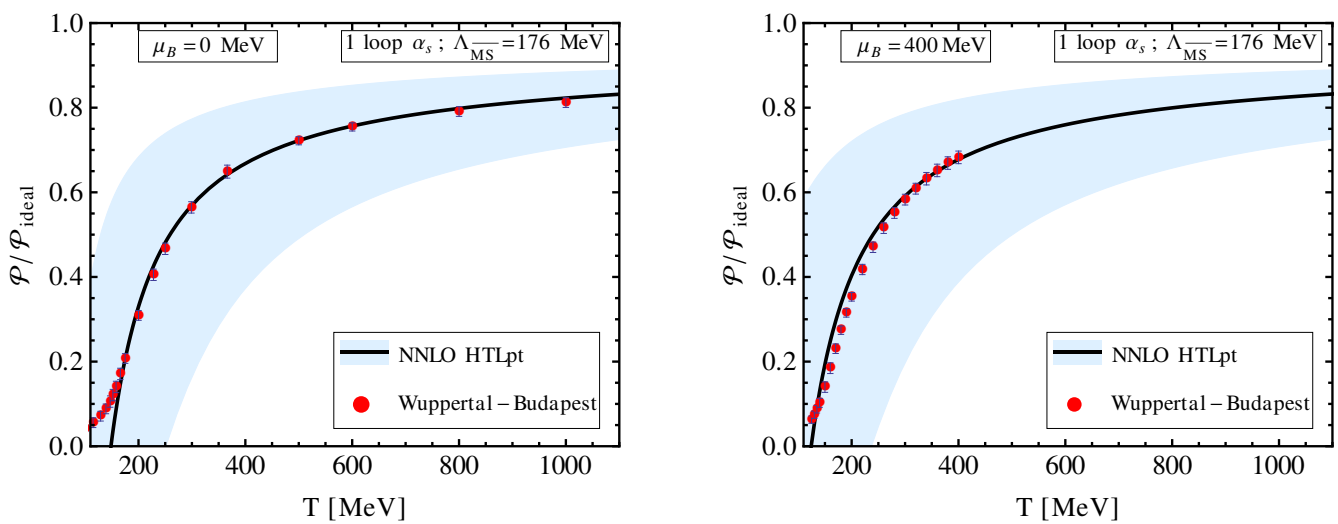

Figure 1: Comparison of NNLO HTLpt pressure with lattice data from Borsányi et al. [21] and [22] at $\mu_{B}=0$ (left) and $\mu_{B}=400 \mathrm{MeV}$ (right), respectively.

In Fig. 1 we compare our NNLO HTLpt pressure scaled with ideal gas pressure for $\mu_{B}=0$ (left) and $\mu_{B}=400 \mathrm{MeV}$ (right) with lattice data. In Fig. 2 we compare the NNLO HTLpt energy
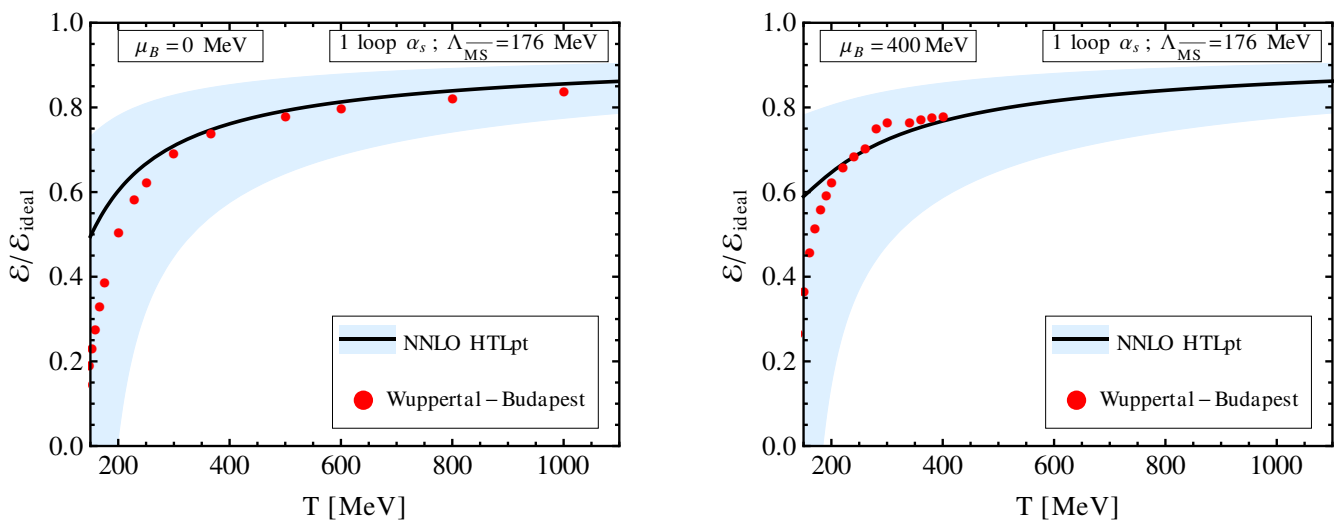

Figure 2: Comparison of the $\mu_{B}=0$ (left) and $\mu_{B}=400 \mathrm{MeV}$ (right) NNLO HTLpt energy density with lattice data from Borsányi et al. [21] and [22], respectively.

density scaled with ideal gas energy density for $\mu_{B}=0$ (left) and $\mu_{B}=400 \mathrm{MeV}$ (right) with available lattice data. In Fig. 3 we compare the NNLO HTLpt trace anomaly scaled with $T^{4}$ for $\mu_{B}=0$ (left) and $\mu_{B}=400 \mathrm{MeV}$ (right) with available lattice data.

In Fig. 4 we compare second-order (left) and fourth-order (right) baryon number susceptibilities scaled with corresponding ideal value with various lattice data. In Fig. 5 we compare the scaled NNLO HTLpt fourth-order diagonal single quark number susceptibility (left) and the only non-vanishing fourth-order off-diagonal quark number susceptibility (right) with various lattice 

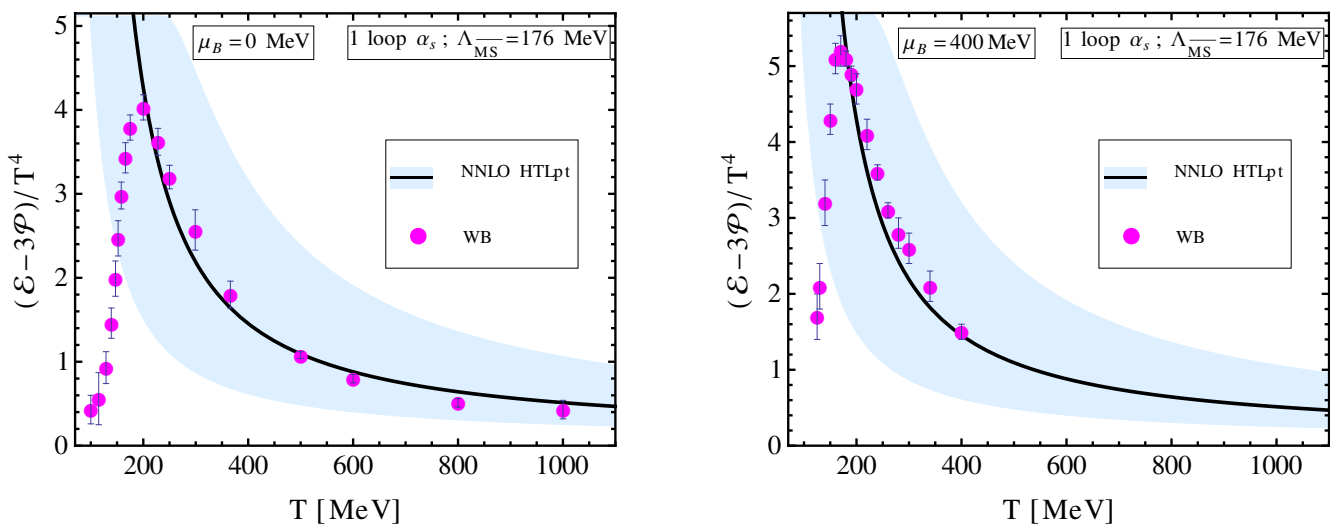

Figure 3: Comparison of the $\mu_{B}=0$ (left) and $\mu_{B}=400 \mathrm{MeV}$ (right) NNLO HTLpt trace anomaly with lattice data from Borsányi et al. [21] and [22], respectively.
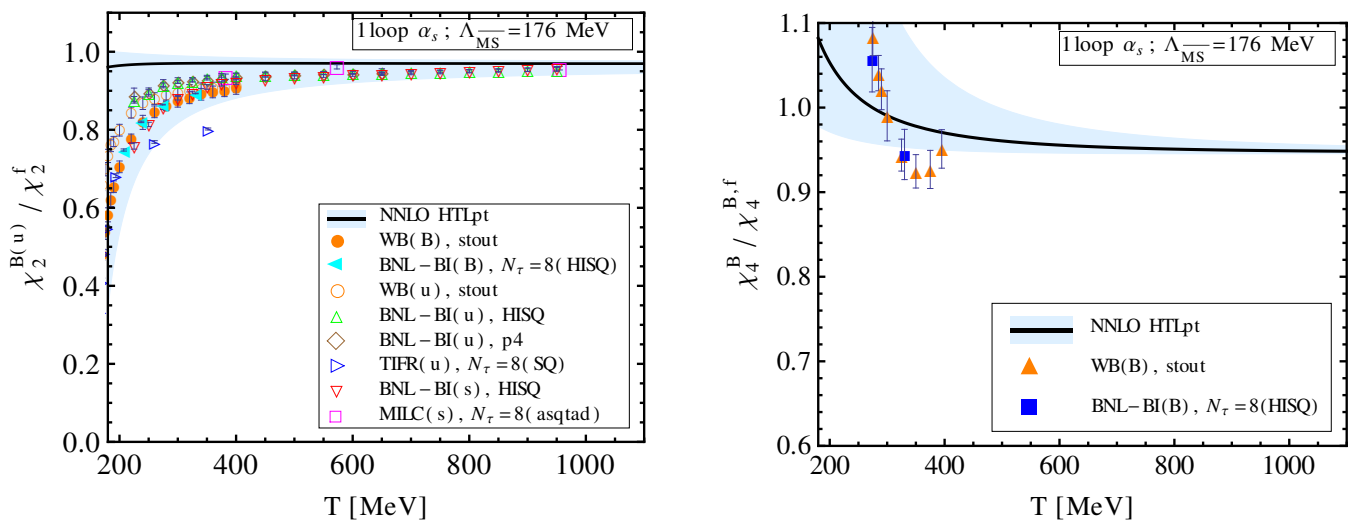

Figure 4: The scaled second-order (left) and fourth-order (right) baryon number susceptibilities compared with various lattice data. The lattice data labeled WB, BNL-BI(B), BNL-BI(u,s), MILC, and TIFR come from Refs. [23], [24], [25], [26], and [27], respectively.

data. As can be seen from Figs. 1-5, our NNLO HTLpt result has quite good agreement with available lattice data. For other quantities such as the entropy density, speed of sound, higher order susceptibilities, etc. see Ref. [19].

\section{Conclusions}

The results for the QCD thermodynamic functions at finite temperature and chemical potential at NNLO in HTLpt have been presented in this proceedings. We have used the final result for thermodynamic functions from Ref. [19] and final result therein is completely analytic, gauge-invariant and does not contain any free fit parameters besides the choice of the renormalization scales $\Lambda_{g}$ and $\Lambda$. As can be seen from Figs. 1-5, the NNLO results have quite reasonable agreement with available lattice data. Since the NNLO HTLpt results for various thermodynamic quantities are in good agreement with lattice data down to temperatures that are relevant for LHC, it offers some hope 

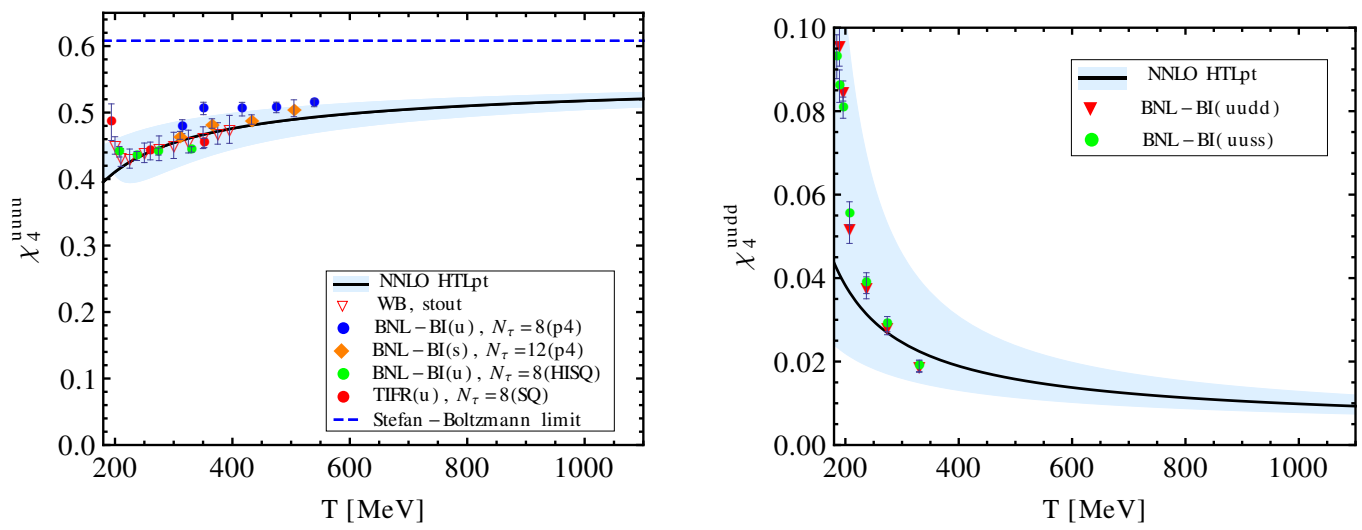

Figure 5: Comparison of the NNLO HTLpt fourth order diagonal single quark number susceptibility (left) and the only non-vanishing fourth order off-diagonal quark number susceptibility (right) with lattice data. In the left figure the dashed blue line indicates the Stefan-Boltzmann limit for this quantity. The data labeled BNL-BI(uudd), BNL-BI(u,s), BNL-BI(uuss), and TIFR come from Refs. [24], [25], [28], and [27], respectively.

that application of HTLpt to the computation of other relevant quantities is not misguided at these temperatures.

\section{References}

[1] K. Kajantie, M. Laine, K. Rummukainen and Y. Schroder, Phys. Rev. D 67, 105008 (2003).

[2] A. Vuorinen, Phys. Rev. D 68, 054017 (2003).

[3] F. Karsch, A. Patkos and P. Petreczky, Phys. Lett. B 401, 69 (1997).

[4] S. Chiku and T. Hatsuda, Phys. Rev. D 58, 076001 (1998).

[5] J. O. Andersen, E. Braaten and M. Strickland, Phys. Rev. D 63, 105008 (2001).

[6] J. O. Andersen and M. Strickland, Phys. Rev. D 64, 105012 (2001).

[7] J. O. Andersen, E. Braaten, and M. Strickland, Phys. Rev. Lett. 83, 2139 (1999).

[8] E. Braaten and R. D. Pisarski, Nucl. Phys. B 337, 569 (1990).

[9] J. O. Andersen, E. Braaten, and M. Strickland, Phys. Rev. D 61, 074016 (2000).

[10] N. Haque, M. G. Mustafa and M. H. Thoma, Phys. Rev. D 84, 054009 (2011).

[11] S. Mogliacci, J. O. Andersen, M. Strickland, N. Su and A. Vuorinen, JHEP 1312, 055 (2013).

[12] J. O. Andersen, E. Braaten, E. Petitgirard, and M. Strickland, Phys. Rev. D 66 (2002) 085016.

[13] J. O. Andersen, E. Petitgirard, and M. Strickland, Phys. Rev. D 70, 045001 (2004).

[14] N. Haque, M. G. Mustafa, and M. Strickland, Phys. Rev. D 87, 105007 (2013), ibid. JHEP 1307, 184 (2013).

[15] N. Su., J. O. Andersen, and M. Strickland, Phys. Rev. Lett. 104, 122003 (2010).

[16] J. O. Andersen, M. Strickland, and N. Su, JHEP 1008, 113 (2010). 
[17] J. O. Andersen, L.E. Leganger, M. Strickland and N. Su, Phys. Lett. B 696, 468 (2011), ibid. JHEP 1108, 053 (2011).

[18] N. Haque, J. O. Andersen, M. G. Mustafa, M. Strickland, and N. Su, Phys. Rev. D 89, 061701 (2014).

[19] N. Haque, A. Bandyopadhyay, J. O. Andersen, M. G. Mustafa, M. Strickland and N. Su, JHEP 1405, 027 (2014).

[20] A. Bazavov, N. Brambilla, X. Garcia i Tormo, P. Petreczky, J. Soto and A. Vairo, Phys. Rev. D 86, 114031 (2012).

[21] S. Borsányi et al., JHEP 1011, 077 (2010).

[22] S. Borsányi et al., JHEP 08, 053 (2012).

[23] S. Borsányi, Z. Fodor, S. D. Katz, S. Krieg, C. Ratti and K. Szabo, JHEP 1201, 138 (2012)

[24] A. Bazavov et al., Phys. Rev. Lett. 111, 082301 (2013).

[25] A. Bazavov et al., arXiv:1309.2317 [hep-lat].

[26] C. Bernard et al., Phys. Rev. D 71, 034504 (2005).

[27] S. Datta, R. V. Gavai and S. Gupta, PoS LATTICE 2013, 202 (2014).

[28] A. Bazavov et al., Phys. Rev. Lett. 109, 192302 (2012). 